\title{
"Concordance between comorbidity data from patient self-report interviews and medical record documentation" William Corser*1, Alla Sikorskii ${ }^{1}$, Ade Olomu ${ }^{2}$, Manfred Stommel ${ }^{1}$, Camille Proden ${ }^{3}$ and Margaret Holmes-Rovner ${ }^{1,4}$
}

\author{
Address: ${ }^{1}$ College of Nursing, Michigan State University, B500A West Fee Hall., East Lansing, Michigan 48824, USA, ${ }^{2}$ College of Human Medicine, \\ Michigan State University, B338 Clinical Center, East Lansing, Michigan 48824, USA, ${ }^{3}$ Emergent BioSoultions, 3500 N. Martin Luther King Blvd., \\ Lansing, Michigan 48906, USA and ${ }^{4}$ Center for Ethics and Humanities in the Life Sciences, Michigan State University, C208 East Fee Hall, East \\ Lansing, Michigan 48824, USA \\ Email: William Corser* - bill.corser@ht.msu.edu; Alla Sikorskii - alla.sikorskii@hc.msu.edu; Ade Olomu - ade.olomu@ht.msu.edu; \\ Manfred Stommel - manfred.stommel@hc.msu.edu; Camille Proden - prodenc@ebsi.com; Margaret Holmes-Rovner - mholmes@msu.edu \\ * Corresponding author
}

Published: 16 April 2008

BMC Health Services Research 2008, 8:85 doi:10.1186/1472-6963-8-85

This article is available from: http://www.biomedcentral.com/I472-6963/8/85

(C) 2008 Corser et al; licensee BioMed Central Ltd.

This is an Open Access article distributed under the terms of the Creative Commons Attribution License (http://creativecommons.org/licenses/by/2.0), which permits unrestricted use, distribution, and reproduction in any medium, provided the original work is properly cited.
Received: 25 August 2007

Accepted: 16 April 2008

\begin{abstract}
Background: Comorbidity is an important adjustment measure in research focusing on outcomes such as health status and mortality. One recurrent methodological issue concerns the concordance of comorbidity data obtained from different reporting sources. The purpose of these prospectively planned analyses was to examine the concordance of comorbidity data obtained from patient selfreport survey interviews and hospital medical record documentation.
\end{abstract}

Methods: Comorbidity data were obtained using survey interviews and medical record entries from 525 hospitalized Acute Coronary Syndrome patients. Frequencies and descriptive statistics of individual and composite comorbidity data from both sources were completed. Individual item agreement was evaluated with simple and weighted kappas, Spearman Rho coefficients for composite scores.

Results: On average, patients reported more comorbidities during their patient survey interviews (mean $=1.78, \mathrm{SD}=1.99)$ than providers had documented in medical records (mean $=1.27, \mathrm{SD}=$ I.43). Higher proportions of positive responses were obtained from self-reports compared to medical records for all conditions except congestive heart failure and renal disease. Older age and higher depressive symptom levels were significantly associated with poorer levels of data concordance.

Conclusion: These results demonstrate that survey comorbidity data from ACS patients may not be entirely concordat with medical record documentation. In the absence of a gold standard, it is possible that hospital records did not include all pre-admission comorbidities and these patient survey interview methods may need to be refined. Self-report methods to facilitate some patients' complete recall of comorbid conditions may need to be refined by health services researchers.

Trial Registration: ClinicalTrials.gov NCT004I6026. 


\section{Background}

Many researchers have demonstrated the influence of different combinations of comorbid conditions on patient outcomes such as quality of life, depression, and death [1$4]$. While the phenomenon of composite comorbidity is considered a complex function of the number and severity of pre-existing health conditions a hospital patient possesses, there is currently no gold standard to measure their composite comorbidity $[5,6]$.

Although health services researchers have debated the specific causal pathways of different comorbidity combinations on health outcomes, researchers have less frequently tested the concordance of comorbidity data obtained from various reporting sources, [5-8] calling for further investigation [2,5,7-11]. Providers and researchers need to identify the more rigorous collection methods concerning patient-level comorbidity data for the formulation of patients' treatment plans and research concerning their subsequent health outcomes $[2,5,8,9]$.

In the literature, researchers have identified three primary sources for obtaining patient-level comorbidity data: a) administrative diagnosis databases or clinical patient registries, $[12,13]$ b) medical records, $[9,10]$ and c) patient self-reports [11,14-17]. Due to the frequently missing data in many administrative diagnosis databases, experts have suggested that patient medical records may be a more complete source of comorbidity data $[2,5,14,16]$.

Still, medical records possess limitations related to: a) inconsistent or absent documentation standards, b) limited availability of recent documentation, and c) underreporting of pre-admission conditions judged by providers to be less pertinent to patients' admitting diagnoses $[10,11,15]$. Medical record notes also often contain elements of both patient self-report and earlier provider documentation, sometimes offering a hybrid source of original data $[10,11,18]$.

\section{Medical Record Source}

One of the most frequently advocated methods used to generate comorbidity data from medical records is the Charlson Comorbidity Index (CCI), $[19,20]$ which has been tested with large samples in numerous settings $[1,5,6,8]$. A patient's composite CCI score is calculated as a weighted sum of the presence of 19 documented health conditions such as Congestive Heart Failure, Diabetes, or Peripheral Vascular Disease. The CCI method was originally developed and tested with a sample of 607 medical patients, with weights assigned to different conditions derived from relative risk estimates of 30-day breast cancer mortality obtained from proportional hazards models [19]. Researchers have obtained similar predictive results concerning patient complications and functional out- comes for cardiac, diabetes, and depression samples $[1,4,6,8]$.

\section{Patient Self-Report Source}

As an alternative to medical records, patient may be asked about their comorbidities in mailed surveys or individual telephone or face-to-face interviews $[9,11,14,21]$. The primary difference from medical records is that survey methods result in "unfiltered" self-reports, (e.g. self-reports that are not filtered through the additional questioning of healthcare personnel). Researchers have demonstrated patient self-reports to be a generally reliable data source through careful rewordings of more complex individual comorbidity items $[9-11,14,17,21]$.

In 1996, Katz, et al. [20] tested a self-report comorbidity source method comprised of a brief questionnaire which includes the same overall series of comorbidity items as the CCI $[19,20]$. Initially, hospital patients were asked if they had experienced each health condition before their current admission, with some questions reworded using more recognizable diagnosis terms (e.g. Chronic Pulmonary Disease converted into Asthma, Emphysema, and Chronic Bronchitis, or Acute Myocardial Infarction (AMI) reworded to Heart Attack). Several additional questions are asked whether patients take medications for certain conditions to prompt patients' recall [21]. The individual item weightings for the Katz [21] method are almost entirely the same as for the CCI $[19,20]$.

\section{Comparison of $\mathrm{CCl}$ and Katz Comorbidity Data Patterns}

In the original Katz et al study, test-retest reliability coefficients were assessed by intra-class correlation, with estimates of 0.91 obtained from the self-report questionnaire compared to 0.92 for the CCI method [21]. Spearman correlation coefficients between composite scores from the two sources were somewhat weak at 0.63 , and even smaller for less-educated patients. In 1996, Katz et al estimated the cost of obtaining each patient's comorbidity data to be $\$ 0.93$ dollars per mailed questionnaire, $\$ 1.67$ per self-report interview, versus $\$ 3.50$ per medical record audit [21].

Individual condition data obtained concerning diagnoses such as Diabetes, Hypertension, Stroke, and Cancer from various self-reporting forms have demonstrated consistently higher levels of concordance $[9,10,22]$. Other conditions such as Heart Failure, Arthritic Conditions, or Pulmonary Conditions have beenless concordant in elderly or veteran patient samples $[9,10,22]$. Some studies have failed to show that socio-demographic characteristics such as increased age, marital status, or completed education levels exert significant influences on concordance levels between the reporting methods $[10,15]$. Other studies have found that younger age, female gender, and/or more 
completed education significantly increased concordance levels $[9,22]$.

\section{Purpose Statement}

The purpose of the prospectively planned study analyses was to examine the concordance levels between comorbidity data generated from Acute Coronary Syndrome (ACS) patients using two related self-report and medical record sources. Rather than treating either source as the gold standard, the authors examined the relative concordance between these two methods and patient predictors affecting concordance levels. The following research questions were addressed:

1. "What is the level of concordant validity of individual item responses and composite comorbidity scores obtained from ACS patient self-report interviews and medical record documentation sources?"

2. "Which patient characteristics predict discordance between comorbidity data obtained from ACS patient self-report interviews and medical record documentation?"

Before the analyses, the authors had hypothesized that the self-report source would provide more complete data concerning certain comorbid conditions affecting patients' daily post-discharge health and function Due to the specific ACS admitting diagnosis under which patients were admitted, the authors also anticipated that medical record notes would reflect a general underreporting of conditions assessed by hospital providers to be less pertinent to patients' cardiac diagnoses. Based on the findings of earlier groups, $[10,22]$ they further expected that the examination of data concordance from ACS patients would provide a more homogenous sampling framework than using data from patients admitted with multiple diagnoses or comorbid conditions.

\section{Methods}

\section{Sample}

The primary randomized intervention study from which these data were obtained enrolled a total sample of 719 ACS patients who had been hospitalized in five midMichigan hospitals [23]. Study inclusion criteria included a working admission diagnosis of ACS, age $\geq 21$ years, possession of a functional home telephone, and documented serum Troponin I levels $\geq$ established upper normal limits during index hospitalization. Exclusion criteria were: inability to speak English and/or complete phone interviews, or discharge to a non-home setting. Nurse recruiters recruited patients between January 14, 2002 and April 13, 2003. The results of this primary study have already been published [4,24-27].
Data regarding patients' comorbidities were collected after discharge from both: a) structured baseline telephone patient interviews $(\mathrm{N}=525)$ using the Katz [21] selfreport method, and $\mathrm{b}$ ) hospital medical record audits ( $\mathrm{N}$ $=710$ ) using the original CCI $[19,20]$ method with paper or electronic medical records (approximately 50\% in each form). Baseline interviews were conducted after index hospitalization (Mean $=14.11$ days, $\mathrm{SD}=9.6$ ), by trained interviewers at a Michigan State University survey institute using a structured telephone interview protocol. Before any patient data were collected, approval had been obtained from the institutional review boards on campus and each participating hospital. Data collectors were blinded to patients' study group status.

All medical record data were collected by a group of study nurses who had been oriented to the chart auditing manual and protocols by the study Community Project Manager. Comorbidity data were obtained from medical record: 1 . face sheets, 2 . history and physical reports, and 3. admission and discharge notes. Chart auditors entered data onto a standard data collection form and referred to the chart audit manual concerning specific comorbidity data fields. Additional string field data were entered for several rare health conditions not included in the CCI $[19,20]$ framework. Periodic chart auditor meetings were conducted to review chart audit protocols, and the results of redundant chart audits were reviewed by the community project manager to confirm the overall data entry reliability of newer auditors. The Community Project Manager continued to sample each reviewer's charts to maintain quality control. Reliability $\geq 98 \%$ was maintained throughout. Errors were largely omitting items in the history and physical, with little bearing on the study data set. This high level of inter-rater reliability is higher that many studies using the CCI $[19,20]$ method. Interclass correlation coefficients of standardized chart audits typically range from 0.83 to 0.93 , likely due to consistent supervision in our study $[28,29]$.

Patient socio-demographic data collected by interview included: patient age category, gender, race (categorized into White or Non-White), and education completed (categorized into less than high school, high school graduate, or at least some college or more). Clinical measures included: a) level of pre-admission physical activity from the Activity Status Index, [30] and b) level of pre-admission depressive symptoms from the Center for Epidemiologic Studies-Depression (CESD) [31].

\section{Data Set Characteristics}

Of the total of 719 enrolled patients, 525 (73.0\% of enrolled) patients completed a post-discharge baseline interview to offer almost complete self-report sociodemographic and comorbidity data. Patients who con- 
sented, but did not participate in the baseline interview were more likely to have received anti-anxiety medications (OR: 2.58, p < 0.01), and were more likely to be minorities using US census categories (OR: 2.02, p < 0.01 ). Fifteen post-discharge deaths were identified from State of Michigan vital records; mortality was not a major contributor to attrition.

Less than $1 \%$ of the baseline interviewed patients refused to answer any comorbidity item, or indicated that they were unsure whether they possessed a condition. Only comorbidity data documented in one of the primary medical record locations were included in these analyses. No distinction was made between missing or refused data obtained from either source. Concordance analyses were limited to the 525 sample patients who provided comorbidity data from both reporting sources.

\section{Data Concerns}

Due to our ACS working admission diagnosis inclusion criterion, all patients were documented as have recently sustained an AMI. Still, due to patients' recent cardiac admission possibly biasing their recall of having sustained an AMI, composite comorbidity scores were evaluated from each source with and without this item included.

\section{Analyses}

Descriptive summary statistics were used to describe the comorbidity data from both reporting sources. Composite comorbidity scores from both sources were calculated, and compared using matched t-tests. In addition, Spearman Rho coefficients between composite comorbidity scores were estimated.
To analyze concordance between individual comorbidity items, we calculated the proportion of total agreement (on both reported presence and absence), and kappa coefficients with 95\% confidence intervals for each condition. McNemar's tests $[32,33]$ were used to test the concordance between the two data sources. Finally, a series of multivariate logistic regression models were run using age, gender, completed education, and depressive symptoms as predictors of agreement or disagreement in reporting patterns from the two methods for individual comorbidity items [34]. Due to the inadequate cell frequencies, the race variable was not included in the models.

The logistic regression models evaluated the probability of comorbidities appearing in either one source but not the other versus the agreement of both sources. The logistic regression analysis was conducted with adjustment for clustering of patients within hospitals using a generalized estimating equations approach with a compound symmetry correlation matrix $[35,36]$. All statistical tests were twosided and performed using S.A.S. version 9.1 [37].

\section{Results}

Study recruiters completed a mean number of 2.44 (SD 1.87) contacts with 1,707 patients who had been initially assessed by recruiters to be eligible, with 988 of these patients either refusing to participate ( 419 or $24.5 \%$ ) or assessed to be too sick to enroll (569 or $33.3 \%$ ). Table 1 lists the major socio-demographic and clinical characteristics of the 525 patients who provided comorbidity data from both reporting sources.

Table I: Demographic \& Clinical Characteristics of Patients at Baseline $(n=525)$

\begin{tabular}{|c|c|c|}
\hline Variable & $\mathbf{N}$ & \\
\hline Age at admission & 525 & $\mathbf{M}=\mathbf{5 9 . 7 3}(\mathrm{SD} 12.00)$ \\
\hline Gender & 525 & \\
\hline Male & & $334(63.6 \%)$ \\
\hline Female & & 191 (36.4\%) \\
\hline White/Non-White Race & 525 & \\
\hline White & & $443(84.4 \%)$ \\
\hline Non-White/Multiracial/Other & & $82(15.6 \%)$ \\
\hline Current Marital Status & 525 & \\
\hline Married & & $350(66.7 \%)$ \\
\hline Divorced/Separated/Widowed & & $174(33.1 \%)$ \\
\hline Work for Pay of Profit? & 524 & \\
\hline Yes & & $226(43.0 \%)$ \\
\hline No & & $298(56.8 \%)$ \\
\hline Completed Education & 521 & \\
\hline Less than High School & & 99 (18.9\%) \\
\hline High school diploma & & $196(37.3 \%)$ \\
\hline Some college or more & & $230(43.8 \%)$ \\
\hline Family Income & 467 & \\
\hline Less than $\$ 15,000$ per year & & I I 3 (25.0\%) \\
\hline$\$ 15,000$ or more per year & & 354 (75.8\%) \\
\hline Activity Status Index [26] (scale $0-54.55)$ & 525 & $M=29.56(S D \mid 7.21)$ \\
\hline CESD Depression [27] (scale 0 - 60) & 524 & $M=13.56(S D \mid 0.48)$ \\
\hline Ejection Fraction & 452 & $\mathbf{M}=\mathbf{5 0 . 1 9}(\mathrm{SD} 12.93)$ \\
\hline
\end{tabular}


Only $132(25 \%)$ patients had a documented ejection fraction of less than 45\%. Notably, although 154 (29.3\% of total sample) of patients reported having sustained a heart attack during their baseline interviews, only $126(81.8 \%$ of these same patients) had a prior AMI documented in their medical records.

Table 2 lists the descriptive statistics of the composite scores obtained from the two reporting sources, their quartiles, and Spearman coefficients. The magnitude of Spearman correlation coefficient with and without AMI included reflected disagreement of the two sources that was confirmed by the comparison of the mean composite scores. The means were significantly higher for the selfreport method $(\mathrm{p}<.01)$, both with and without the AMI item included. The effect sizes for the difference in means between the two methods were .31 with AMI included, and .29 with AMI excluded. According to Cohen's classification, [38] these effect sizes are considered medium. Therefore, highly statistically significant disagreement was not simply due to large sample size, but reflected a practically important difference.

Table 3 displays the patterns of individual comorbidity item data derived from the two sources, as well as Kappa coefficients, percents of overall agreement, and p-values of McNemar's test [31,32] levels of agreement. Only two comorbid conditions were more frequently reported in patient medical records: Congestive Heart Failure/Heart Failure $(\mathrm{p}<.01)$ and Renal/Kidney Disease $(p=.13)$. All other conditions were more frequently reported by patients than documented in the medical records, with the differences in reporting rates reaching statistical significance for all conditions except Peripheral Vascular Disease and Diabetes with End-organ Damage. Figure 1 displays rates of agreement and disagreement between the two sources. With exception of AMI, the kappa agreement coefficients reflected fair to poor agreement between comorbidity data obtained from the two sources [38].

Tables 4 lists the odds ratios of disagreement between the two reporting sources versus their agreement for individual comorbidity items with prevalence of at least 3\% in each data source. The reported odds ratios are adjusted for age, depressive symptoms, and level of completed educa- tion. Since gender had an insignificant association with the concordance of any individual comorbidity items, it was not included in the final model. For many comorbid conditions, increased age was significantly associated with an increased probability of disagreement between the two sources.

The odds of medical record documentation of diabetes and renal disease when the patient him/herself does not report these conditions increased with age (with respective odds ratios of 2.59 and 2.16 relative to agreement for each additional 10 years of age). At the same time, the odds of a patient reporting diabetes, a prior heart attack, congestive heart failure, cerebrovascular disease and cancer not documented in the medical record also increases with age. Only in the case of diabetes do we see both types of disagreement increasing with age.

The effect of depressive symptoms was somewhat similar to age with higher symptom scores associated with a higher probability of disagreement. This association reached statistical significance for increased patient selfreports of cerebrovascular disease, chronic pulmonary disease, and cancer. The effect of completed education reached statistical significance for two conditions, but did not show a consistent direction of effect (the magnitude of the odds ratios was very different across multiple items).

\section{Discussion}

These results indicate that the concordance of ACS patient comorbidity data from patient survey interviews and medical record documentation sources may certainly vary by condition and patient characteristics. The distinct cardiac nature of these ACS patients' clinical encounters may have biased both patient and provider reports, since certain conditions such as chronic pulmonary disease or congestive heart failure were more likely assessed by providers during each patient's hospital admission.

Our original hypothesis that medical records data would reflect a general underreporting of certain conditions that providers considered to be less pertinent to patients' ACS admitting diagnoses appears to be generally supported. Our hypothesis that non-cardiac conditions would be

Table 2: Composite Comorbidity Score Data Patterns

\begin{tabular}{|c|c|c|c|c|c|c|}
\hline Variable & Mean (St Dev) & Median & QI & Q3 & Spearman rho & $\mathbf{T}(\mathbf{P})$ \\
\hline Charlson $[18,19]$ composite score (prior AMI included) * & $1.27(1.43)$ & I & 0 & 2 & $0.57(\mathrm{p}<.01)$ & $7.12(<.01)$ \\
\hline Katz [20] composite score (prior AMI included) $*$ & $1.78(1.99)$ & 1 & 0 & 3 & & \\
\hline Charlson $[18,19]$ composite score (prior AMI excluded) & $1.03(1.30)$ & I & 0 & 2 & $0.50(p<.0 I)$ & $6.73(<.01)$ \\
\hline Katz [20] composite score (prior AMI excluded) & $1.49(1.82)$ & 1 & 0 & 2 & & \\
\hline
\end{tabular}

* (possible range of 0 (i.e. no comorbid conditions) to 37 (maximum possible number of comorbid conditions) 
Table 3: Individual Comorbidity Item Agreement

\begin{tabular}{|c|c|c|c|c|c|}
\hline Comorbid Condition & $\begin{array}{l}\text { Charlson Medical } \\
\text { Record N Yes (\%) }\end{array}$ & $\begin{array}{c}\text { Katz Self-report N } \\
\text { Yes (\%) }\end{array}$ & Kappa (95\% CI) & $\begin{array}{l}\text { Overall Percent } \\
\text { Agreement* }\end{array}$ & $\begin{array}{c}\text { P-Value of } \\
\text { McNemar's test }\end{array}$ \\
\hline Prior AMI/Heart attack & $126(24)$ & I54 (29.33) & $0.63(0.56,0.70)$ & 86 & $<0.01$ \\
\hline Congestive heart failure & $150(28.6)$ & $95(18.1)$ & $0.09(0.01,0.18)$ & 67 & $<.01$ \\
\hline $\begin{array}{l}\text { Peripheral vascular } \\
\text { disease }\end{array}$ & $28(5.3)$ & $37(7.1)$ & $0.43(0.27,0.58)$ & 93 & 0.13 \\
\hline Cerebrovascular disease & $31(5.9)$ & $56(10.7)$ & $0.54(0.4 \mathrm{I}, 0.67)$ & 93 & $<0.01$ \\
\hline $\begin{array}{l}\text { Chronic pulmonary } \\
\text { Disease/Asthma } \\
\text { bronchitis }\end{array}$ & $45(8.6)$ & $59(11.2)$ & $0.43(0.30,0.55)$ & 90 & 0.06 \\
\hline Renal/Kidney disease & $24(4.6)$ & $17(3.2)$ & $0.47(0.28,0.66)$ & 96 & 0.13 \\
\hline Diabetes & $116(22.1)$ & $142(27.1)$ & $0.80(0.72,0.86)$ & 92 & $<0.01$ \\
\hline $\begin{array}{l}\text { Diabetes with end-organ } \\
\text { damage }\end{array}$ & $23(4.4)$ & $26(5.0)$ & $0.42(0.24,0.60)$ & 95 & 0.56 \\
\hline Hemiparesis & $3(0.6)$ & $24(4.6)$ & $0.14(-0.04,0.32)$ & 96 & $<0.01$ \\
\hline Ulcer disease & $30(5.7)$ & $74(14.1)$ & $0.29(0.17,0.41)$ & 87 & $<0.01$ \\
\hline $\begin{array}{l}\text { Connective Tissue } \\
\text { Disorder/Arthritis- } \\
\text { Rheum. Arthritis }\end{array}$ & $5(1.0)$ & $92(17.5)$ & $0.07(0.00,0.13)$ & 83 & $<0.01$ \\
\hline Dementia & $0(0)$ & $4(0.8)$ & & & \\
\hline Any type of cancer & $26(5.0)$ & $44(8.4)$ & $0.33(0.18,0.48)$ & 92 & $<.01$ \\
\hline $\begin{array}{l}\text { Liver Disease (mild to } \\
\text { moderate) }\end{array}$ & I (0.2) & $3(0.6)$ & & 99 & \\
\hline
\end{tabular}

* overall percent agreement was defined as the number of concordant counts (both answered "yes" or both answered "no" in two sources) divided by the total sample size and expressed as a percent.

underreported by providers due to patients' admission diagnoses does appear to be supported if we accept patient self-reports as more comprehensive.

These findings specifically demonstrate that for patients who are older or have depressive symptoms, the agreement between data obtained from the two reporting sources may also be significantly affected. Although the practice of including family members could introduce potential response biases, having family members serve as co-informants during research interviews may help older and/or depressed patients recall a large proportion of their earlier diagnosed conditions to improve reporting.

We could conclude similar to earlier studies $[10,22]$ that patients with smaller numbers of self-perceived conditions to recall during interviews may have reported a larger proportion of their total documented comorbidities to increase concordance with medical records. Although the generalizability of these results to non-cardiac or higher comorbidity samples may be limited, our results still suggest that discordance levels between these two sources may be even higher for sicker patient groups with multiple admission diagnoses.

The apparent influence of patients' level of completed education was inconsistent across different conditions. For example, Table 4 indicates that better educated patients are less likely to report a prior AMI or congestive heart failure that is not in the medical record, but are more likely to report cancer that is not documented. This may well be an indication that better educated patients offered more reliable information, if we are willing to accept the assumption that the medical records were more accurate on cardiac-related diseases, but not on less relevant diseases such as cancer. Wording refinements during selfreport interviews of some technical medical terms still in the Katz [21] method may facilitate more accurate responses from patients with lower health literacy or education.

Our analyses may have been affected by several limitations. The majority of these post-discharge data came from a sample of hospitalized ACS patients with relatively few reported comorbidities in a specific area of the Midwest. Our use of hospital medical records may have limited our ability to capture the entirely of patients' earlier documented conditions. Wording differences between the self-report source (i.e. before your hospitalization) and medical record source (developed to capture both current and past conditions) items may account for some discordance. Since we lacked self-report comorbidity data from non-baseline interviewed patients, we were unable to compare self-reporting patterns of interviewed and noninterviewed sample subgroups. 


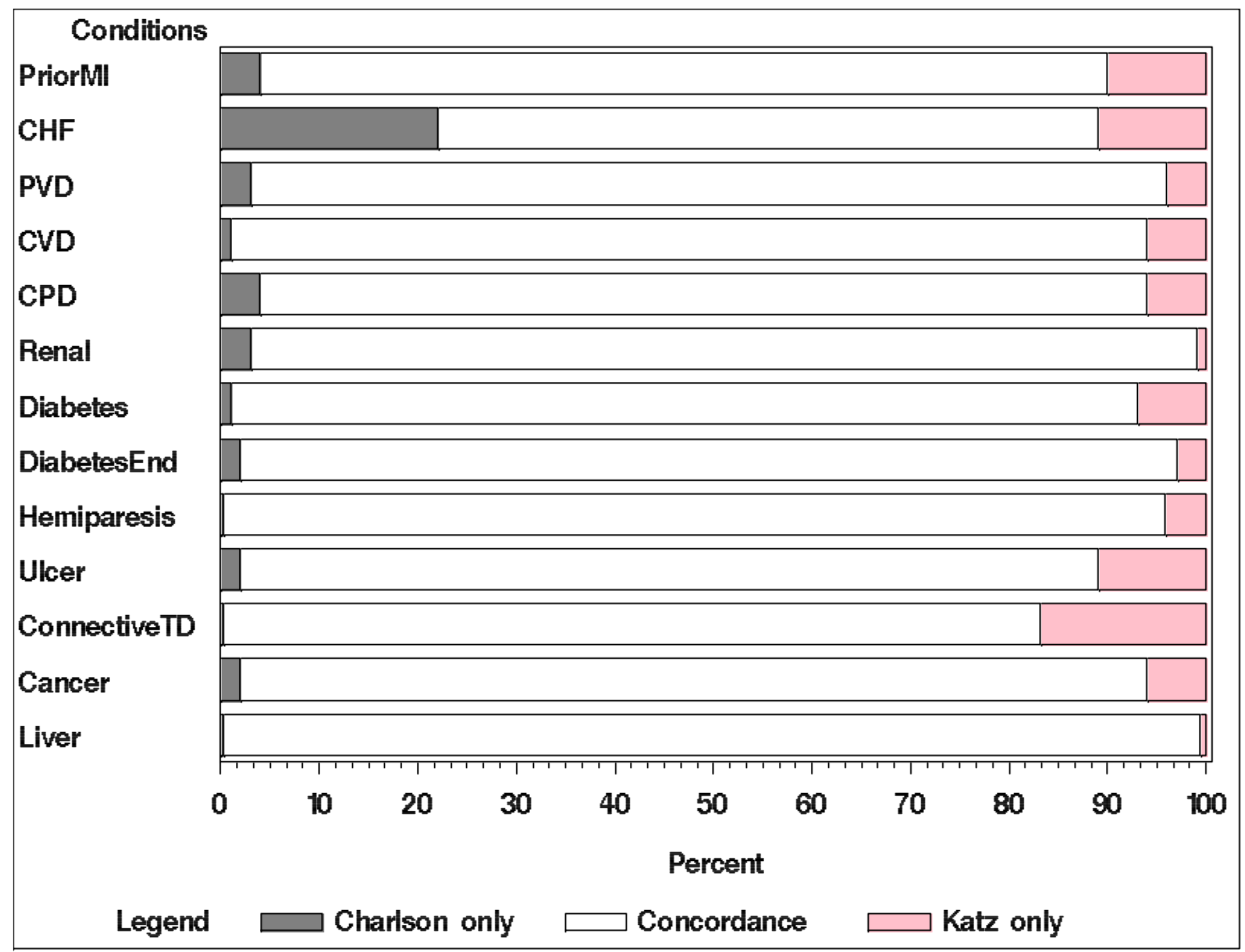

Figure I

Rates of agreement and disagreement of individual comorbidity items from two data sources. NOTE: the relative proportions of comorbid conditions vary.

\section{Conclusion}

Similar to several earlier studies, [2-4,17] our findings strongly suggest that using patient self-report comorbidity reporting sources may be preferable for conditions more likely to affect patients' routine quality of life and functional status (e.g. connective tissue disorders, arthritic conditions, diabetes, stomach ailments). For these conditions, the gold standard measurement issue remains problematic in most studies for the prediction of patient health outcomes. Several research groups have suggested, however, that the parallel use of comorbidity data collection methods from both sources may be necessary to analyze or predict some types of health outcomes $[5,9,11,14,18]$. Although the data provided by patients' significant others or the use of carefully worded comorbidity checklists may be subject to response biases, such methods may help prompt increased recall of past or longstanding comorbid conditions to augment providers' hospitalization documentation.

Additional testing of the factors influencing relative concordance between patient and provider comorbidity data sources may enable researchers to develop more rigorous comorbidity data reporting methods, particularly for heavily comorbid patients. These results support the conclusion made by earlier researchers that further adjustments in comorbidity data collection methods will be required to obtain the fullest comorbidity data to validly predict patient health outcomes $[2,4,11,16]$.

Due to a variety of patient and setting-level factors, studies testing the influences of comorbidities on subsequent 
Table 4: Odds Ratios (ORs) and 95\% confidence intervals for ORs of comorbid conditions documented in medical record but not in patient self report or in self-report but not documented (relative to agreement of both sources): effects of patient age, education and depressive symptoms.

\begin{tabular}{|c|c|c|c|c|}
\hline Comorbid Condition & Presence in & Age & $\begin{array}{c}\text { Education }>=\text { High school versus } \\
<\text { High school }\end{array}$ & CES-D \\
\hline \multirow[t]{2}{*}{ Prior AMI } & medical record but not in self report & $1.03(0.99,1.06)$ & $1.37^{* * * *}(0.44,4.32)$ & $1.03(1.00,1.07)$ \\
\hline & self report but not in medical record & $1.03(1.01,1.06)^{* *}$ & $0.46(0.24,0.89)^{*}$ & $1.01(0.98,1.04)$ \\
\hline \multirow[t]{2}{*}{ Congestive heart failure } & medical record but not in self report & $1.01(0.99,1.02)$ & $0.62(0.36,1.06)$ & $1.00(0.98,1.02)$ \\
\hline & self report but not in medical record & $1.03(1.01,1.05)^{*}$ & $0.44(0.23,0.84)^{*}$ & $1.03(1.00,1.05)$ \\
\hline \multirow[t]{2}{*}{ Peripheral vascular disease } & medical record but not in self report & $1.02(1.98,1.07)$ & $0.42(0.13,1.41)$ & $1.01(0.96,1.07)$ \\
\hline & self report but not in medical record & $1.01(0.98,1.05)$ & $1.02(0.32,3.20)$ & $0.99(0.95,1.04)$ \\
\hline \multirow[t]{2}{*}{ Cerebrovascular disease } & medical record but not in self report & $1.04(0.97,1.11)$ & $0.57(0.09,3.48)$ & $1.01(0.93,1.09)$ \\
\hline & self report but not in medical record & $\mathrm{I} .04(\mathrm{I} .0 \mathrm{I}, \mathrm{I} .07)^{*}$ & $1.14(0.46,2.84)$ & $1.04(1.01,1.08)^{*}$ \\
\hline \multirow[t]{2}{*}{ Chronic pulmonary disease } & medical record but not in self report & $1.01(0.98,1.05)$ & $0.47(0.17,1.27)$ & $1.01(0.97,1.06)$ \\
\hline & self report but not in medical record & $1.01(0.98,1.04)$ & $1.31(0.5 \mathrm{I}, 3.38)$ & $1.04(1.00,1.07)^{*}$ \\
\hline \multirow[t]{2}{*}{ Renal disease } & medical record but not in self report & $1.10(1.05,1.15)^{* *}$ & $2.56(0.50,12.97)$ & $1.01(0.95,1.07)$ \\
\hline & self report but not in medical record & $1.03(0.97,1.09)$ & $0.22(0.04,1.08)$ & $1.02(0.96,1.10)$ \\
\hline \multirow[t]{2}{*}{ Diabetes } & medical record but not in self report & $1.08(1.02,1.16)^{*}$ & $0.65(0.13,3.20)$ & $1.08(1.01,1.15)^{*}$ \\
\hline & self report but not in medical record & $1.04(1.01,1.07)^{* *}$ & $1.47(0.57,3.83)$ & $1.03(1.00,1.07)$ \\
\hline \multirow{2}{*}{$\begin{array}{l}\text { Diabetes with end-organ } \\
\text { damage }\end{array}$} & medical record but not in self report & $1.08(1.03,1.14)^{* *}$ & $0.84(0.23,3.09)$ & $1.06(1.01,1.12)^{*}$ \\
\hline & self report but not in medical record & $1.05(1.00,1.09)^{*}$ & $0.47(0.16,1.45)$ & $1.04(0.99,1.09)$ \\
\hline \multirow[t]{2}{*}{ Ulcer disease } & medical record but not in self report & $1.01(0.96,1.06)$ & $0.68(0.17,2.72)$ & $1.00(0,95,1.06)$ \\
\hline & self report but not in medical record & $1.02(1.00,1.05)$ & $0.79(0.40,1.56)$ & $1.02(0.99,1.04)$ \\
\hline \multirow[t]{2}{*}{ Any type of cancer } & medical record but not in self report & $1.01(0.96,1.05)$ & $0.57(0.16,2.02)$ & $1.01(0.96,1.06)$ \\
\hline & self report but not in medical record & $1.06(1.03,1.09)^{* *}$ & $3.72(1.05,3.12)^{*}$ & $1.04(1.01,1.08)^{*}$ \\
\hline
\end{tabular}

*** $p<.01$

$*_{p}<.05$

*** For example, $O R=1.37$ for the level education reflects the fact that the odds of prior AMI documented in medical record but not in self report relative to being documented in both sources were $37 \%$ higher for those with at least high school education compared to those with less than high school education.

patient outcomes will continue to be complex. Judging from our results, additional testing of reporting methods to generate more accurate comorbidity data will be required for distinct patient subgroups. Although these findings indicate that patient self-report methods may provide a feasible source of ACS patient data for some comorbid conditions, the factors contributing to discordance between reporting sources as we have identified will need to be more fully investigated for future outcomes research.

\section{Abbreviations}

ACS: Acute Coronary Syndrome; AMI : Acute Myocardial Infarction; CCI: Charlson Comorbidity Index.

\section{Availability and requirements}

\section{Clinical Trials URL: http://clinicaltrials.gov}

\section{Competing interests}

The author(s) declare that they have no competing interests.

\section{Authors' contributions}

WC conception and design, drafting and revising manuscript. AS conception and design, analysis and interpretation of data, revising manuscript. AO conception and design, revising manuscript. MS conception and design, revising manuscript. PP acquisition of study data. MH-R conception and design, revising manuscript.

\section{Acknowledgements}

Special thanks to database manager Chrystal Price, MS, for data entry, and Fuli Gao, MS for data analysis assistance.

This study was supported in part by an AHRQ ROI grant (HSI053I), "Translating Research: Patient Decision Support/Coaching." (Dr. Margaret Holmes-Rovner, Principal Investigator).

\section{References}

I. Tuominen U, Blom M, Hirvonen I, Seitsalo S, Lehto M, Paavolainen P, Hietanien K, Rissanen P, Sintonen H: The effect of co-morbidities on health-related quality of life in patients placed on the waiting list for total joint replacement. Health Qual Life Outcomes 2007, 5:16.

2. Bayliss EA, Ellis JL, Steiner JF: Subjective Assessments of Comorbidity correlate with Quality of Life Health Outcomes: Initial Validation of a Comorbidity Assessment. Health Qual Life Outcomes 2005, 3:5I-59.

3. Corser WD: An investigation of patient outcomes related to comorbidity and interdisciplinary hospital discharge planning. Outcomes Mngmt 2004, 8:45-5I. 
4. Olomu AB, Corser WD, Holmes-Rovner MM: Self-report Comorbidity data and functional outcomes in acute coronary syndrome patients. (published abstract) 28th National Annual SGIM Meeting: New Orleans, LA. J Gen Intern Med 2005, 20(Suppl I):72.

5. Lash TL, Mor V, Wieland D, Ferrucci L, Satariano W, Silliman RA: Methodology, design, and analytic techniques to address measurement of comorbid disease. J Gerontol 2007, 62:28I-285.

6. Gijsen R, Hoeymans N, Schellevis FG, Ruwaard D, Satariano WA, Geertrudis AMV: Causes and consequences of comorbidity: a review. J Clin Epid 200I, 54:66I-674.

7. Kraemer HC: Statistical Issues in Assessing Comorbidity. Stats in Med 1995, 14:72I-733.

8. Roos LL, Stranc L, James RC, Li J: Complications, comorbidities, and mortality: improving classification and prediction. Health Serv Res 1997, 32:229-238.

9. Simpson CF, Boyd CM, Carlson MC, Griswold ME, Guralnik JM, Fried LP: Agreement between self-report of disease diagnoses and medical record validation in disabled older women: factors that modify agreement. J Am Geriatr Soc 2004, 52:123-127.

10. Skinner KM, Miller DR, Lincoln E, Lee A, Kazis LE: Concordance between respondent self-reports and medical records for chronic conditions: experience from the veterans health study. Ambulatory Care Manage 2005, 28: 102-1 I0.

II. Tisnado DM, Adams JL, Liu H, Damberg CL, Chen WP, Hu FA, Carlisle DM, Mangione CM, Kahn K: What is the concordance between the medical record and patient self-report as data sources for ambulatory care. Med Care 2006, 44: I32-140.

12. Schneeweiss S, Maclure M: Use of comorbidity scores for control of confounding in studies using administrative databases. Int J Epidemiol 2000, 29:89I-898.

13. Humphries KH, Rankin JM, Carere RG, Buller CE, Kiely FM, Spinelli JJ: Comorbidity data in outcomes research: are clinical data derived from administrative databases a reliable alternative to chart review? I Clin Epidemiol 2000, 53:343-349.

14. Ingram SS, Seo PH, Martell RE, Clipp EC, Doyle ME, Montoya GS, Cohen HJ: Comprehensive assessment of the elderly cancer patient: the feasibility of self-report methodology. I Clin Oncol 2002, 20:770-775.

15. Sangha O, Stucki G, Liang MH, Fossel AH, Katz JN: The self-administered comorbidity questionnaire: a new method to assess comorbidity for clinical and health services research. Arthritis Rheum Res 2003, 49(2): I56-163.

16. DeGroot V, Beckerman H, Lankhorst G], Bouter LM: How to measure comorbidity: a critical review of available methods. J Clin Epidemiol 2003, 56:22I-229.

17. Selim AJ, Fincke G, Ren XS, Lee A, Rogers WH, Miller DR, Skinner KM, Linzer M, Kazis LE: Comorbidity assessments based on patient report: results from the veterans health study. Ambul Care Manage 2004, 27:28I-295.

18. Byles JE, D'Este C, Parkinson L, O'Connell R, Treloar C: Single index of multimorbidity did not predict multiple outcomes. J Clin Epidemiol 2005, 58:997-1005.

19. Charlson ME, Pompei P, Ales KL, MacKenzie CR: A new method of classifying prognostic comorbidity in longitudinal studies: development and validation. J Chron Dis 1987, 40:373-383.

20. Charlson M, Szatrowski TP, Peterson J, Gold J: Validation of a combined comorbidity index. J Clin Epidem 1994, 47: I245- I25।.

21. Katz JN, Chang LC, Sangha O, Fossel AH, Bates DW: Can comorbidity be measured by questionnaire rather than medical record review? Med Care 1996, 34:73-84.

22. Okura Y, Urban LH, Mahoney DW, Jacobsen SJ, Rodeheffer RJ: Agreement between self-report questionnaires and medical record data substantial for diabetes, hypertension, myocardial infarction, and stroke, but not for heart failure. J Clin Epidemiol 2004, 57:1096-1 103.

23. Holmes-Rovner M, Stommel M, Corser W, Olomu A, Holtrop J, Siddiqi A, Dunn SI: Does outpatient telephone coaching add to hospital quality improvement? (under review). J Gen Intern Med.

24. Holtrop JS, Corser WD, Spence-Jones G, Brooks G, Holmes-Rovner M, Stommel M: Health behavior goals of cardiac patients after hospitalization. Amer J Health Behav 2006, 30:387-399.

25. Dunn SL, Corser WD, Stommel M, Holmes-Rovner M: Hopelessness and depression in the early recovery period after hospitalization for acute coronary syndrome. I Cardiopulm Rehab 2006, 26: 152-159.
26. Stommel M, Olomu A, Holmes-Rovner M, Corser W: Changes in practice patterns affecting in-hospital and post-discharge survival among ACS patients. BioMedCrtl-Health Ser Res 2006, 6:140.

27. Yang Z, Olomu A, Corser WD, Holmes-Rovner M: Outpatient medication use \& health outcomes in post acute coronary syndrome patients. Amer J Mnged Care 2006, I 2( I 0):58I-7.

28. Hall SF, Groome PA, Streiner DL, Rochen PA: Inter-rater reliability of measurements of comorbid illness should be reported. J Clin Epidemiol 2006, 59:926-933.

29. Bernadini J, Callen S, Fried L, Piraino B: Inter-rater reliability and annual rescoring of the Charlson Comorbidity Index. Adv Perit Dial 2004, 20: I25-I27.

30. Hlatky MA, Boineau RE, Higginbotham MB, Lee KL, Mark DB, Califf RM, Cobb FR, Pryor DB: A brief self-administered questionnaire to determine functional capacity. Am J Card 1989, 64:65I-654.

31. Devins G: Center for Epidemiologic Studies Depression Scale. Test Crit 1985, 2:144-160

32. Agresti A: Categorical Data Analysis. New York:John Wiley \& Sons, Inc; 1999.

33. McNemar Q: Note on the sampling error of the difference between correlated proportions or percentages. Psychometrika 1947, 12:153-157.

34. Hosmer DW, Lemeshow S: Applied Logistic Regression. 2nd edition. New York: Wiley; 2000.

35. Liang KY, Zeger SL: Longitudinal data analysis using generalized linear models. Biometrika |986, 73:| 3-22.

36. Zeger SL, Liang KY: Longitudinal data analysis for discrete and continuous outcomes. Biometrics 1986, 42:12I-I 30.

37. SAS Institute Inc: S.A.S. Software, version 9.I. In (computer program) Cary NC.

38. Cohen J: Statistical Power Analysis for the Behavioral Sciences. 2nd edition. New York: Academic Press; 1988.

\section{Pre-publication history}

The pre-publication history for this paper can be accessed here:

http://www.biomedcentral.com/1472-6963/8/85/prepub
Publish with Bio Med Central and every scientist can read your work free of charge

"BioMed Central will be the most significant development for disseminating the results of biomedical research in our lifetime. "

Sir Paul Nurse, Cancer Research UK

Your research papers will be:

- available free of charge to the entire biomedical community

- peer reviewed and published immediately upon acceptance

- cited in PubMed and archived on PubMed Central

- yours - you keep the copyright
BioMedcentral 